\title{
Supercritical fluid synthesis and possible properties of "cubic graphite"
}

\author{
A. V. Pokropivny ${ }^{1,2}$, A. N. Enyashin ${ }^{3}$, A. S. Smolyar ${ }^{1,4}$, V. A. Kuts ${ }^{5}$, V. G. Gurin ${ }^{5}$, \\ S. A. Antipov ${ }^{5}$, P. M. Silenko ${ }^{1}$, Yu. M. Solonin ${ }^{1}$ \\ ${ }^{1}$ Frantsevich Institute for Problems of Materials Science NASU, Kyiv, Ukraine \\ ${ }^{2}$ G. V. Kurdyumov Institute for Metal Physics, NASU, Kyiv, Ukraine \\ ${ }^{3}$ Institute of Solid State Chemistry UB RAS, Ekaterinburg, Russia \\ ${ }^{4}$ M.P. Semenenko Institute of Geochemistry, Mineralogy and Ore Formation NASU, Kyiv, Ukraine \\ ${ }^{5}$ Institute of Magnetism NASU, Kyiv, Ukraine \\ apokr@ukr.net, enyashin@ihim.uran.ru
}

PACS 81.10.Dn, 61.66.Bi, 61.48.+c, 61.50.Ah

DOI 10.17586/2220-8054-2020-11-4-408-416

\begin{abstract}
We report on supercritical fluid synthesis of an intermediate carbon phase - austite - at a pressure of $180 \mathrm{MPa}$ and temperatures $500-700^{\circ} \mathrm{C}$ from soot as a precursor and supercritical carbon dioxide as a solvent. According to the results of electron and X-ray diffractions, spectral measurements and density-functional theory calculations, the observed carbon phase is proved to be cubic with a lattice parameter value of $8.96 \pm 0.05 \AA$ and a possible structural type as for KFI zeolite.
\end{abstract}

Keywords: crystal growth, carbon allotropes, electron microscopy, X-ray diffraction, DFT calculations.

Received: 15 June 2020

Revised: 10 July 2020

\section{Introduction}

Supercritical fluid synthesis is a common method for the high-pressure high-temperature synthesis of different oxides, sulfides, nitrides, carbon phases as well as self-assembling of nanomaterials [1,2]. Different gases can be employed for creating pressure during supercritical fluid synthesis as an inert medium (He, Ar) or a solvent and a chemical reagent $\left(\mathrm{H}_{2}, \mathrm{~N}_{2}, \mathrm{H}_{2} \mathrm{O}, \mathrm{CO}_{2}\right.$ etc.). The dissolving capacity of fluids is rapidly increased with increasing of the pressure, which is also important for supercritical fluid extraction. On the other hand, the velocity of crystallization in supercritical medium is increased up to $0.1-0.3 \mathrm{~mm} / \mathrm{h}$, which is at least five times greater than for hydrothermal methods. Moreover, crystals formed from the gas phases may have a high degree of perfection, in comparison with other methods.

In 1963 Aust and Drickamer claimed on synthesis of a new cubic carbon phase (henceforth, austite) by transformation from single crystal of graphite at $15 \mathrm{GPa}$ and $180 \mathrm{~K}$ [3]. There was a sharp rise of resistance with pressure at this point, accompanied by drifting upward with time - a behavior typical of a sluggish first-order phase transition. The mixture of new phase with graphite was found to have the density of $2.35-2.4 \mathrm{~g} / \mathrm{cm}^{3}$ with the float-sink tests. $\mathrm{X}$-ray diffraction analysis of the partially transformed mixture showed that this phase could be cubic with the lattice parameter of $5.545 \AA$, having 24 atoms per unit cell and a crystal density of $\sim 2.8 \mathrm{~g} / \mathrm{cm}^{3}$ [3].

In 1967 Bandy reported, that Drickamer rejected on the facts of the synthesis of austite in a private communication [4]. Therefore, the discovery of a new phase in carbon phase diagram was not accepted by the scientific community. It should be noted that authors [3] had not published official refutation of their work. Presumably the indisputable authority of Bandy misled other scientists, who could confirm or refute completely their extraordinary results. As a result, in 1974 the pattern of this phase (No 18-311) was deleted from the database. Nevertheless, at the moment several forms of diamond, nanotubes, fullerenes, a large number of carbon fullerites, and other allotropic modifications have been discovered on carbon phase diagram, hence, an observation of new carbon phase is not a surprise since the last half of twentieth century [5].

However, the austite-like phases have been repeatedly observed after Aust and Drickamer. Namely, Fedoseev et. al. reproduced an austite-like phase during the growth of diamond from the gas phase [6], by Smolyar et. al. with a supercritical fluid treatment of graphite [7], and, possibly, by Shterenberg et. al. from channel black with a heat treatment at $1250^{\circ} \mathrm{C}$ in the presence of $\mathrm{Ni}$ at $3.7 \mathrm{MPa}$ [8]. The hardness of austite was found to be intermediate, namely 1-5 GPa [7]. Shumilova et. al. discovered austite-like phase in the nature among the products of graphite mineralization [9]. The mineral lignite from Dakota Star Mines [10], the existence of which was questionable, could also be hypothesized as this phase. The structure was interpreted for the first time as a cubic lattice of $\mathrm{C}_{24}$ fullerenes arranged as zeolite-like LTA lattice [11,12]. 
In the last two decades, other new phases of carbon and boron nitride attract outstanding interest of the same or even higher level, as fullerenes and nanotubes do. All of these new materials can be used in the architectures of future devices or, alternatively, as advanced materials with prominent absorption properties or superhardness. Among these new phases as the $\mathrm{C}_{8}$ phase [13], E-phase of boron nitride [14,15] and fulborenite $\mathrm{B}_{12} \mathrm{~N}_{12}$ [16] are remarkable. Crystal structures of these phases were proposed quite recently, although they were synthesized in the last half of the twentieth century. The problem of structure determination and attribution of new forms of carbon and BN is ordinary due to their small mass yields after synthesis, unlike that, for example, nanotubes and fullerenes, and due to low atom weight of constituent elements. Theoretically, several hypothetical zeolite-like carbon structures (ATO, KAN, ATN, AFI etc.) are proposed for future identifications [17-26].

The goal of this work is to obtain a sample of austite phase ("cubic graphite") using the supercritical fluid synthesis from soot and to revise the structural data on the received samples. The density-functional theory methods are employed to supply the results of experiments.

\section{Experimental part}

\subsection{Synthesis and characterization}

Equipment for supercritical fluid synthesis represents the reactor with an inner holder for the samples, aggregates for compressions and cleaning of gas, heating system, and automatic control system. The equipment allows one to create the gas pressures up to $1 \mathrm{GPa}$ for a short time period $(0.5-1 \mathrm{~h})$ and to maintain the pressure during the long time period (hours, days) [1-7].

The soot was used as a precursor and supercritical carbon dioxide as a solvent. It was saturated in the reactor at pressure $95 \mathrm{MPa}$ for 25 hours at ambient temperature. After that, the temperature was cyclically increased and reduced in the intervals of $500-700^{\circ} \mathrm{C}$ during 4 hours at pressure $180 \mathrm{MPa}$. The reaction product was observed as polycrystalline plates and as separate crystals in the soot.

Transformed soot powders were studied using different physical methods. X-ray powder diffraction (XRPD) patterns were received on DRON-2 diffractometer in the range of $2 \theta=5^{\circ}-90^{\circ}$ at $\mathrm{Cu}_{K \alpha}$ radiation. Electron diffraction (ED) patterns were obtained at room temperature with a Philips EM-400T transmission electron microscope, operating at acceleration voltage of $100 \mathrm{kV}$. The samples were prepared by crushing in a mortar and dispersal of the synthesized crystallites in ethanol, and subsequent deposition on a grid containing holes. The elemental composition of the plates of new phase was studied with the JEOL spectrometer.

\subsection{Computational details}

The calculations were performed within the framework of the density-functional theory (DFT) using the SIESTA 4.0 implementation $[27,28]$. The exchange-correlation potential within the Generalized Gradient Approximation (GGA) with the Perdew-Burke-Ernzerhof parametrization was used. The core electrons were treated within the frozen core approximation, applying norm-conserving Troullier-Martins pseudopotentials. The valence electrons for $\mathrm{C}$ were taken as $2 s^{2} 2 p^{2}$. The pseudopotential core radii were chosen, as suggested by Martins, and equal to $1.3 \mathrm{a}_{B}$ for $s-$ and $p$-states of C. In all calculations double- $\zeta$ basis set was used. The k-point mesh was generated by the method of Monkhorst and Pack with a cutoff $10 \AA$ for the k-point sampling. The real-space grid used for the numeric integrations was set to correspond to the energy cutoff of $200 \mathrm{Ry}$. All calculations were performed using variable-cell and atomic position relaxations, with convergence criteria corresponding to the maximum residual stress of $0.1 \mathrm{GPa}$ for each component of the stress tensor, and the maximum residual force component of $0.01 \mathrm{eV} / \AA$.

Apart from the geometry optimization using DFT method, the stability, mechanical and thermal properties were tested using geometry optimization and molecular-dynamics (MD) simulations within the framework of the densityfunctional tight-binding method (DFTB) [29] in $\Gamma$-point approximation as implemented in the deMon software [30]. The structures were MD annealed during $20 \mathrm{ps}$ with the time step of $1.0 \mathrm{fs}$ as canonical (NVT) ensembles at temperatures $T=300$ and $600 \mathrm{~K}$ using global Berendsen thermostat with the time constant $100 \mathrm{fs}$.

The optimized geometry of all carbon allotropes was used to simulate theoretical XRD spectra for wavelength $\lambda=1.5406 \AA$ ( $\mathrm{CuK}_{\alpha}$ radiation). All structures were considered as periodic 3D crystals. The smearing of reflection profiles was approximated with correction for the isotropic atomic temperature factor [31].

\section{Results and Discussion}

\subsection{Electron diffraction analysis}

ED patterns from both untransformed soot and synthesized crystal are collected in Fig. 1. It should be noted that synthesized particles were found to be hard, in comparison with starting material, in good agreement with previous research of this phase [7]. The test samples consisted of crystalline microparticles of 10 to $100 \mu \mathrm{m}$ in size. The 
observed crystals were solid, unlike carbon black, but crumble well in an agate mortar. Therefore, their hardness may be estimated as the one not exceeding 6.5-7.0 units on the Mohs scale. Microparticles of untransformed soot were amorphous or badly-crystalline with the characteristic parameter of $3.6 \AA$, hence, larger than that for graphite. The patterns of the synthesized sample can be attributed without any restriction to a cubic phase with the characteristic lattice parameter $8.96 \pm 0.05 \AA$.

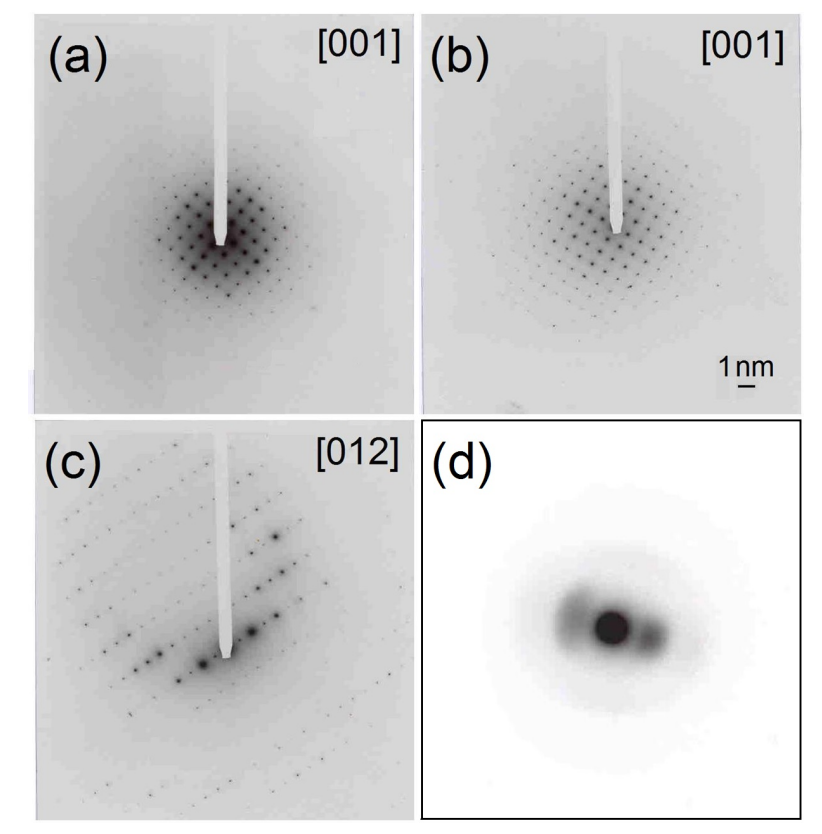

FIG. 1. Electron diffractograms in different projections (a-c) for the carbon samples prepared using supercritical fluid synthesis and in comparison to the diffractogram for the carbon precursor (d). Common scale bar is given in (b)

Qualitative analysis of the elemental composition using TEM spectrometer reveals only carbon, and, possibly, light elements in the samples under investigations. Using spectral quantitative analysis at the surface of the polycrystal plates, the composition was found to be 98-99 at\% of carbon, with the 0.5-1 at\% of oxygen and much less then $1 \%$ of other elements. Thus, the phase is really a pure carbon, while oxygen is likely adsorbed on the surface, and the rest elements are impurities arising from the reactor and from the sample preparation for the spectrometer.

\subsection{X-ray diffraction analysis}

Results of XRPD analysis of the sample of powders are listed in column 1 of Table 1 . For comparison, the theoretical XRD reflections of austite have been calculated with the JSV computer code for the structure proposed as carbon KFI zeolite [32] with the space group Im-3m (column 2 of Table 1). The $h k l$ indices, $d h k l$-spacings (prior to brackets), relative intensities (in brackets) and recalculated lattice parameters (after brackets) are tabulated. Interpretation of reciprocal spaces clearly indicates the presence of a single cubic phase of $8.95 \pm 0.15 \AA$, in excellent agreement with the results of electron diffraction. Simultaneously, the lattice parameter of the austite sample from [3] (column 3 of Table 1) can be found slightly greater, which may be related to the difference in sample preparation. Rietveld refinements are not possible because the powders of new phase are pyrogenetically bound to graphite or some other form of carbon. This is not the only problem associated with carbon or boron nitride phases.

Column 2 of Table 1 shows the results of model powder diffraction for zeolite-like KFI carbon with the lattice constant $a=8.95 \AA$. The calculated diffraction patterns from the proposed KFI structure correlate well with those from our sample and from report of Aust et al. [3]. There are (200), (222) and (330) main calculated reflexes for the proposed structure. Experimentally, two of them are presented for our sample in good agreement with the calculations. Though, main experimental reflexes (110), (200), (220), (332) have only weak analogs of theoretical reflexes as calculated for zeolite-like KFI carbon. The synthesized phase should have a texture along (220) and (110) planes, since their two reflexes rank first in intensity. The (222) strong reflex is not observed by Aust et al. [3], but it is observed from our samples. And vice versa (422) reflex is observed by Aust et al. [3], but it is not observed from our sample. 
TABLE 1. Theoretically derived as for carbon KFI zeolite (column 2), and experimental XRPD patterns (column 3 and 4) with corresponding lattice parameters (in $\AA$ after brackets). Subscripts in column 1 denote the formerly ascribed indices for this phase [3]. X-ray diffraction pattern of the experimental samples contains additionally several peaks of different intensities, which correspond to other phases

\begin{tabular}{|c|c|c|c|}
\hline$h k l$ & Theoretical KFI structure for $a=8.95 \AA$ & Experimental in our work & Experimental [3] \\
\hline 100 & & $9.10(5), a=9.10$ & \\
\hline 110 & $6.329(6)$ & $6.27(30), a=8.87$ & \\
\hline 111 & & $5.30(5), a=9.17$ & \\
\hline 200 & $4.475(100)$ & $4.42(15), a=8.84$ & \\
\hline $210_{(110)}$ & & $3.95(10), a=8.83$ & \\
\hline 211 & $3.654(<1)$ & $3.61(10), a=8.84$ & \\
\hline $220_{(111)}$ & $3.164(3)$ & $3.17(100), a=8.96$ & $3.208(10), a=9.07$ \\
\hline $310_{(200)}$ & $2.830(10)$ & $2.80(10), a=8.85$ & $2.770(50), a=8.76$ \\
\hline 222 & $2.584(41)$ & $2.55(10), a=8.83$ & $2.467(50), a=8.90$ \\
\hline 321 & $2.392(<1)$ & $2.365(10), a=8.85$ & \\
\hline $400_{(211)}$ & $2.238(3)$ & $2.214(10), a=8.86$ & \\
\hline 330,411 & $2.110(25)$ & & \\
\hline 420 & $2.001(5)$ & $2.00(20), a=8.94$ & \\
\hline 332 & $1.908(5)$ & $1.932(30), a=9.06$ & $1.961(10), a=8.99$ \\
\hline $421_{(220)}$ & & $1.932(30), a=8.85$ & \\
\hline $422_{(221)}$ & $1.827(4)$ & & $1.844(10), a=9.03$ \\
\hline 510,431 & $1.755(<1)$ & & \\
\hline 521 & $1.634(1)$ & & \\
\hline $440_{(222)}$ & $1.582(1)$ & $1.589(5), a=8.99$ & $1.600(10), a=9.05$ \\
\hline 433,530 & $1.535(3)$ & & \\
\hline $442,600_{(321)}$ & $1.492(<1)$ & & $1.485(10), a=8.91$ \\
\hline 532 & $1.452(6)$ & & \\
\hline
\end{tabular}

It should be noted here that a set of structural transformations influence on the powder diffraction patterns. The essential differences in peaks distribution exist not only for different method of genesis, but also from probe to probe of the same method. In particular, sample of [3] is more textured with a poor set of diffraction lines in comparison with our sample. Nevertheless, first three XRD peaks can be fairly attributed to the peaks of our sample.

\subsection{DFT computational data}

The preliminary experimental results for observed cubic carbon phase with the lattice parameter $\sim 8.95 \AA$ suggest the model corresponding to zeolite-like KFI lattice, which impose the unit cell consisting of $96 \mathrm{C}$ atoms $-\mathrm{C}_{96}$ (vide infra). Here, we employ several DFT approaches to infix the possible crystal structure of synthesized carbon phase as austite, confirming its dynamical and mechanical stabilities. Apart from the zeolite-like KFI $\mathrm{C}_{96}$, the diamond with face-centered cubic lattice was chosen as a reference system to study structural, electronic and elastic properties. In addition, similar calculations were also performed for other porous carbon $-\mathrm{C}_{24}$ fullerite with simple cubic lattice (or LTA carbon zeolite), where $\mathrm{C}_{24}$ fullerenes are connected by square-like edges [11,12]. While the properties of diamond are profoundly established from both experimental and theoretical views, the $\mathrm{C}_{24}$ fullerite was selected due to its structural relativity to $\mathrm{C}_{96}$ (Fig. 2). The convenient KFI structure of $\mathrm{C}_{96}$ can be represented as a primitive bodycentered cubic lattice of $\mathrm{C}_{48}$ building blocks. The latter have the shape of truncated cuboctahedra and are assembled by means of hexagonal edges creating a characteristic motif of hexagonal prisms. Yet, simple cubic lattice of $\mathrm{C}_{24}$ fullerite may be also represented as composed of the same $\mathrm{C}_{48}$ building blocks linked via square-like edges, which form a primitive face-centered cubic lattice.

The calculated structural, electronic and mechanical properties of diamond have validated the calculational approaches used in this work and demonstrated their fair adequacy for the reproduction of basic properties of carbon 

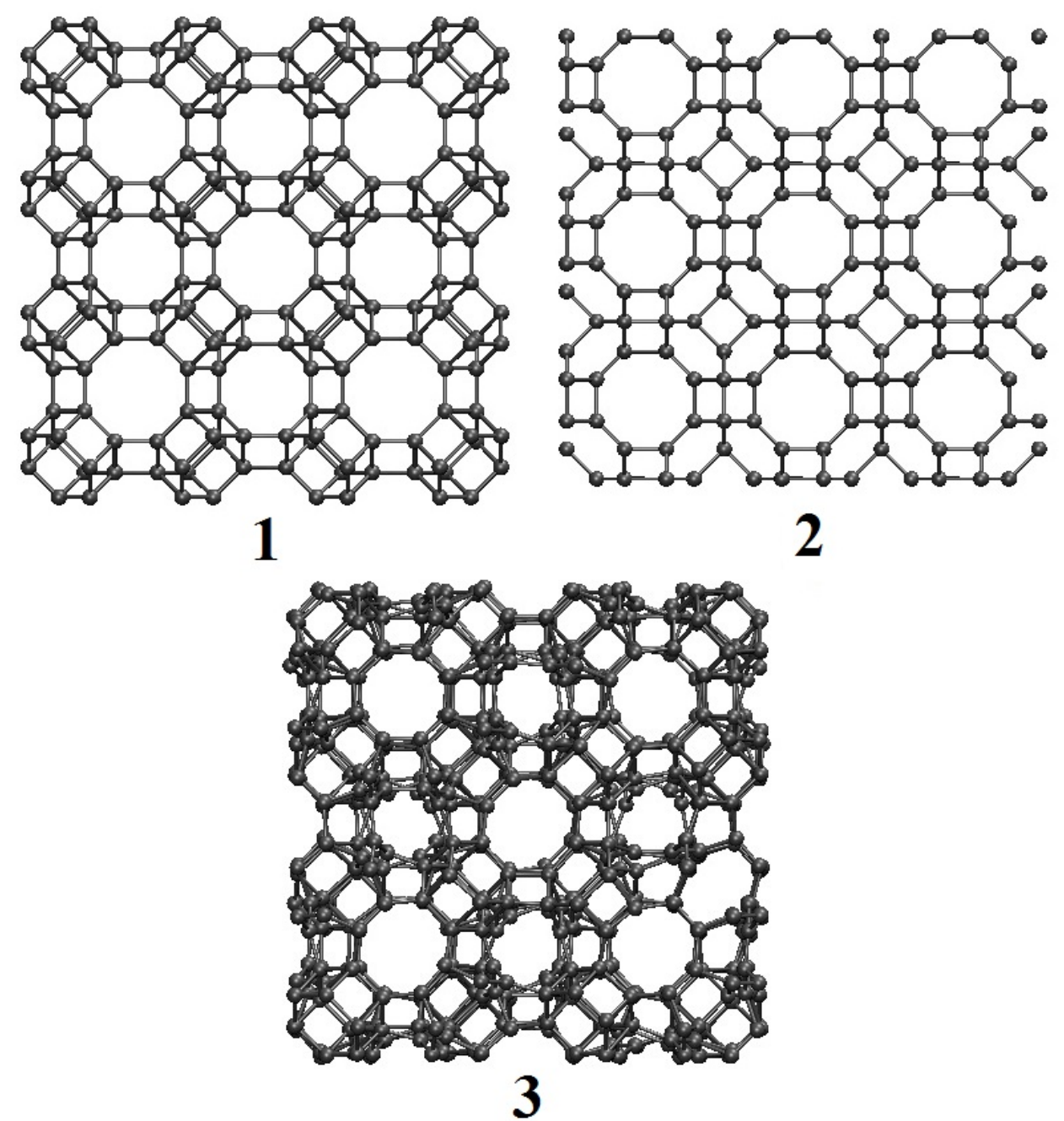

FIG. 2. Structures of subtle carbon allotropes studied in this work: the fragments of austite $\mathrm{C}_{96}$ with zeolite-like KFI lattice (bcc- $\mathrm{C}_{48}$ ) (1) and $\mathrm{C}_{24}$ with zeolite-like LTA lattice (fcc- $\mathrm{C}_{48}$ ) (2) after GGA-DFT geometry optimization; the fragments of austite $\mathrm{C}_{96}$ after DFTB molecular-dynamics simulations at $600 \mathrm{~K}(3)$

allotropes (Table 2). The error in the estimation of lattice parameter does not exceed $0.3 \%$. In agreement with the experimental data, the cubic diamond is characterized as a wide band gap semiconductor with non-direct type of transition from $\Gamma$-point to (2/3) $\Gamma$-X point (Fig. 3). As in many DFT approaches, the value of the band gap obtained after DFT-GGA calculations is underestimated by $\sim 1 \mathrm{eV}$. In turn, DFTB approach typically overestimates the band gap, while the features of the band structure are as well fairly reproduced. The values of the bulk modulus estimated either from total energy curve or calculated from independent elastic constants may be found in the fair agreement with the experimental value (Tables 2 and 3). In general, apart of the band gap estimations, both employed calculational methods yield equivalent results.

The calculations of $\mathrm{C}_{96}$ allotrope were performed using primitive body-centered cubic unit cell, which contains 48 atoms. The lattice of subtle $\mathrm{C}_{96}$ allotrope preserves bcc-structure after the geometry optimization (Fig. 2). Contrary to the diamond, the band structure calculation at DFT-GGA level reveals that $\mathrm{C}_{96}$ is a semiconductor with the smaller and direct band gap of about $2.6 \mathrm{eV}$ (Table 2). However, the partial densities of states at the valence band of both carbon allotropes have dominant $2 p \mathrm{C}$ character.

A highly strained coordination of $s p^{3}$-hybridized carbon atoms in $\mathrm{C}_{96}$ cannot be energy gainful and the total energy calculations evidence the lower stability of $\mathrm{C}_{96}$ carbon comparing to the diamond almost on $\sim 1 \mathrm{eV}$ per $\mathrm{C}$ atom. The first hint at the dynamical stability of this lattice was given using molecular dynamics simulations of the supercell consisting of $16 \mathrm{C}_{48}$ building blocks. A reasonably high stability of $\mathrm{C}_{96}$ allotrope can be revealed in the absence of external pressure and at temperature of $300 \mathrm{~K}$, when no change in the structure can be obtained and the 
TABLE 2. Main characteristics of $\mathrm{C}_{96}$ carbon allotrope (KFI-like lattice), $\mathrm{C}_{24}$ fullerite (LTA-like lattice) and diamond in conventional cubic lattice representation: number of atoms in the unit cell $Z$, relative formation energy $(\Delta E$, in eV/atom), lattice parameter ( $a$, in $\AA$ ), mass density ( $\rho$, in $\left.\mathrm{g} / \mathrm{cm}^{3}\right)$, bulk modulus $(\mathrm{B}, \mathrm{GPa})$ and band gap $\left(E_{g}\right.$, in $\left.\mathrm{eV}\right)$, as calculated at different DFT levels

\begin{tabular}{|c|c|c|c|c|c|c|c|}
\hline & Method & $Z$ & $\Delta E$ & $a$ & $\rho$ & $B$ & $E_{g}$ \\
\hline \multirow{3}{*}{ fcc-diamond } & DFT GGA & \multirow{3}{*}{8} & 0.0000 & 3.58 & 3.47 & 493 & 4.20 \\
\hline & DFTB & & 0.0000 & 3.58 & 3.47 & 487 & 7.73 \\
\hline & exp. [25] & & & 3.57 & 3.52 & 442 & $5.46-5.6$ \\
\hline \multirow{2}{*}{$\begin{array}{c}\text { sc-C } \mathrm{C}_{96} \\
\text { or bcc-- } \mathrm{C}_{48}\end{array}$} & DFT GGA & \multirow{2}{*}{96} & 0.8382 & 9.29 & 2.39 & 285 & 2.61 \\
\hline & DFTB & & 1.0659 & 9.35 & 2.34 & 276 & 7.15 \\
\hline \multirow{2}{*}{$\begin{array}{c}\mathrm{sc}_{-} \mathrm{C}_{24} \\
\text { or fcc- } \mathrm{C}_{48}\end{array}$} & DFT GGA & & 0.7501 & 5.93 & 2.30 & 290 & 2.42 \\
\hline & DFTB & & 0.8892 & 5.95 & 2.27 & 280 & 7.15 \\
\hline
\end{tabular}

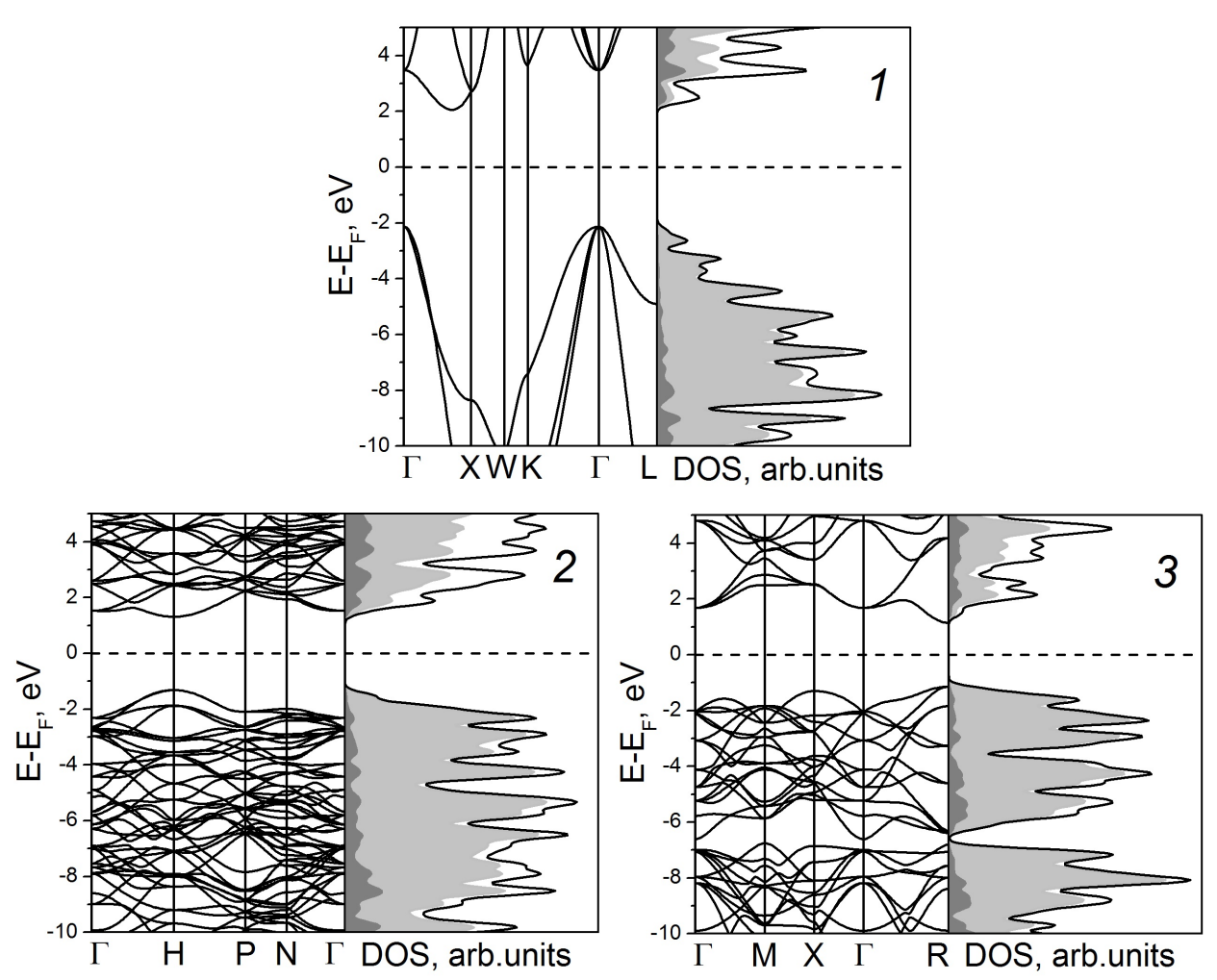

FIG. 3. Band structure and densities of states (DOS) for cubic diamond (1), for austite $\mathrm{C}_{96}$ with zeolite-like KFI lattice (or bcc- $\mathrm{C}_{48}$ ) (2) and for $\mathrm{C}_{24}$ fullerite with zeolite-like LTA lattice (or fcc$\mathrm{C}_{48}$ ) (3) calculated within DFT-GGA approach. $2 \mathrm{pC}$ and $2 \mathrm{sC}$ partial densities of states are painted in light gray and gray, respectively

atom motions are not accompanied with the break of chemical bonds. However, the structure of $\mathrm{C}_{96}$ undergoes a considerable destruction with the partial destruction of hexagonal prisms and occurrence of double $\mathrm{C}=\mathrm{C}$ bonds at $T=600 \mathrm{~K}$ (Fig. 2).

Thereafter, the stability of the lattice was estimated in more details by means of the widely used condition of intrinsic stability, i.e. when the Gibbs free energy of the crystal is in a local minimum with respect to small structural deformations. In terms of elastic constants $\left(C_{i j}\right)$, this stability criterion (so-called mechanical stability) for cubic crystals requires that $\left(C_{11}-C_{12}\right)>0,\left(C_{11}+2 C_{12}\right)>0$, and $C_{44}>0$. In the framework of less sophisticated DFTB method the values of three independent elastic constants $\left(C_{11}, C_{12}\right.$ and $\left.C_{44}\right)$ were evaluated and compared with those of diamond (Table 3). These constants satisfy the aforementioned conditions. Thus, we may assert that the proposed $\mathrm{C}_{96}$ allotrope should be intrinsically stable. 
Calculated elastic constants allow to estimate several other important mechanical properties, which are listed along with available experimental values in Table 3: bulk modulus $B=\left(C_{11}+2 C_{12}\right) / 3$, compressibility $\beta=1 / B$, shear modulus $G=\left(3 C_{44}+C_{11}-C_{12}\right) / 5$, Pugh's indicator $k=G / B$, tetragonal shear moduli $G^{\prime}=1 / 2\left(C_{11}-C_{12}\right)$, Young's modulus $Y=9 B G /(3 B+G)$, Cauchy pressure $C P=C_{12}-C_{44}$, Poisson's ratio $\nu=1 / 2(1-Y / 3 B)$, Zener anisotropy index, $A_{Z}=2 C_{44} /\left(C_{11}-C_{12}\right)$.

The Young's modulus $Y$ of a material is defined as a ratio of linear stress to linear strain, which determines its stiffness. In our study, the Young's modulus of $\mathrm{C}_{96}$ lattice is found to be $Y=475 \mathrm{GPa}$, thus, the material will show a rather high stiffness.

Brittle/ductile behavior is one of many important mechanical characteristics of a material, which is closely related to its reversible compressive deformation and fracture ability. Often, the ductile/brittle nature of a material is related to its Cauchy pressure $C P$ : if $C P$ is negative, the material is expected to be brittle. Another most widely used materials' malleability measure is the Pugh's index $k$ [34]. Namely, at $k<0.57$, a material behaves in a ductile manner, and vice versa, at $k>0.57$, it demonstrates brittleness. According to both indicators (Table 3), $\mathrm{C}_{96}$ carbon allotrope should behave as a brittle material.

Elastic anisotropy of crystals reflects a different bonding character in different directions and has an important implication since it correlates with the possibility to induce microcracks in materials. The elastic anisotropy can be estimated using the Zener's anisotropy index $A_{Z}$ [35]. For isotropic crystals, $A_{Z}=1$, and the deviations of $A_{Z}$ from unity define the extent of elastic anisotropy. From this point of view, $\mathrm{C}_{96}$ allotrope with $A_{Z}=0.65$ should be elastically quite isotropic.

The calculated elastic parameters allow estimate Vickers hardness of the material $\left(H_{V}\right)$ in a few simplified ways. Generally, the hardness of a material is characterized experimentally by indentation and depends strongly on plastic (irreversible) deformation. However, a set of empirical relationships between Vickers hardness and elastic properties was proposed. Herein, the following semi-empirical correlations between Vickers hardness and shear, Young's modulus, and Pugh's index were applied [36,37]: $H_{V}(1)=0.1769 G-2.899 ; H_{V}(2)=0.0608 Y ; H_{V}(3)=2\left(k^{2} G\right)^{0.585}$ -3. Present numerical estimations of $H_{V}$ (Table 3) demonstrate that all three correlations give comparable values and suggest that the $\mathrm{C}_{96}$ allotrope should be a hard material.

As was previously mentioned, $\mathrm{C}_{96}$ or bcc- $\mathrm{C}_{48}$ lattice is related to the lattice of $\mathrm{C}_{24}$ fullerite or fcc- $\mathrm{C}_{48}$. Thus, the simultaneous fabrication of both these carbon allotropes might be expected. In addition, the stability, electronic and mechanical properties were estimated also for $\mathrm{C}_{24}$ fullerite at the same levels of theory (Tables 2 and 3, Fig. 3). Noteworthy, the latter phase characterized earlier by Pokropivny et al $[11,12]$ is slightly more stable, than $\mathrm{C}_{96}$, and posses very similar trends in the mechanical stability of lattice and in the electronic properties. Obviously, the coproduction or the production of individual $\mathrm{C}_{24}$ fullerite under the same experimental conditions of supercritical fluid synthesis cannot be fully excluded.

TABLE 3. Mechanical properties of $s p^{3}$ carbon allotropes as obtained after DFTB calculations: independent elastic constants $\left(C_{i j}\right.$, in GPa), bulk modulus ( $B$, in $\left.\mathrm{GPa}\right)$, compressibility $\left(\beta\right.$, in $\left.\mathrm{GPa}^{-1}\right)$, shear modulus $\left(G\right.$, in GPa), Pugh's indicator $(k)$, tetragonal shear moduli $\left(G^{\prime}\right.$, in GPa), Young's modulus $(Y$, in $\mathrm{GPa})$, Cauchy's pressure $(C P$, in $\mathrm{GPa})$, Poisson's ratio $(\nu)$, Zener's anisotropy index $\left(A_{Z}\right)$, and Vickers hardness $\left(H_{\nu}\right.$, in $\left.\mathrm{GPa}\right)$

\begin{tabular}{|c|c|c|c|c|c|}
\hline System & $C_{11}$ & $C_{12}$ & $C_{44}$ & $B$ & $\beta$ \\
\hline fcc-diamond & $1103.5(1079 *)$ & $178.8(124 *)$ & $602.2(578 *)$ & $487(442 *)$ & 0.0021 \\
\hline sc- $C_{96}\left(b c c-C_{48}\right)$ & 607.0 & 109.7 & 161.7 & 275 & 0.0036 \\
\hline $\mathrm{sc}-\mathrm{C}_{24}\left(\mathrm{fcc}-\mathrm{C}_{48}\right)$ & 550.7 & 145.4 & 259.7 & 281 & 0.0036 \\
\hline System & $G$ & $k$ & $G^{\prime}$ & $Y$ & $C P$ \\
\hline fcc-diamond & 546 & 1.12 & $462(478 *)$ & $1192(1050 *)$ & -423.4 \\
\hline sc- $\mathrm{C}_{96}\left(\mathrm{bcc}-\mathrm{C}_{48}\right)$ & 196 & 0.71 & 249 & 475 & -52.0 \\
\hline $\mathrm{sc}-\mathrm{C}_{24}\left(\mathrm{fcc}-\mathrm{C}_{48}\right)$ & 237 & 0.84 & 203 & 555 & -114.3 \\
\hline System & $\nu$ & $A_{Z}$ & $H_{\nu}(1)$ & $H_{\nu}(2)$ & $H_{\nu}(3)$ \\
\hline fcc-diamond & $0.09(0.1 *)$ & 1.30 & 93.7 & 72.5 & 88.2 \\
\hline sc- $\mathrm{C}_{96}\left(\mathrm{bcc}-\mathrm{C}_{48}\right)$ & 0.21 & 0.65 & 31.8 & 28.9 & 30.8 \\
\hline $\mathrm{sc}-\mathrm{C}_{24}\left(\mathrm{fcc}-\mathrm{C}_{48}\right)$ & 0.17 & 1.28 & 39.0 & 33.7 & 37.0 \\
\hline
\end{tabular}

* experimental values from Ref. [33] 


\section{Summary}

In 1963 Aust and Drickamer [3] found a new phase during transformation of graphite at high pressures. In 2004 a few members of our group offered the structure of this phase on the basis of available data. At that time, the phase was indexed in the cubic system with a period of $\sim 5.55 \AA$ with the structure of LTA zeolite [11,12]. With the support from electron diffraction technique the lattice parameter has been refined and its revised value is found to be approximately $\sim 9 \AA$ instead of $\sim 5.55 \AA$, according to previous experimental data [3] and theoretical [11, 12] reports. The synthesized austite is suggested as carbon phase $\mathrm{C}_{96}$ with zeolite-like KFI lattice (space group Im-3m) in accordance with analysis of electron and X-ray diffraction patterns. As independent method, DFT calculations confirm the mass density, dynamical and mechanical stability of the proposed structure.

In the present experimental work the high level of nucleation of new crystalline carbon phase from disordered material is for the first time observed without additional catalysts, with only supercritical carbon dioxide as a solvent. The presence of graphite and, possibly, chaoite and $\mathrm{C}_{8}$ sodalite phases [13] as concomitant products of synthesis indirectly confirms an assumption that new carbon phases are relatively stable and not rare. Indeed, more then 15 allotropes of carbon have been discovered in nature [38]. Apart from the $\mathrm{C}_{96}$ carbon allotrope with KFI-like structure a wealth of other carbon allotropes might be potentially fabricated under extreme conditions of fluid synthesis. Being fabricated under the conditions far from equilibrium, not all these phases can be hosted at the classical phase diagram of carbon, what can entangle their confident identification and characterization.

While the basic structural, electronic and mechanical properties have been established theoretically, the experimental characterization of newly fabricated carbon $\mathrm{C}_{96}$ phase still remains in its infancy. A set of new synthesis experiments, in particular, under different pressure-temperature and kinetic parameters are planned to increase the yield of cubic carbon and to reveal in details the formation mechanism. The latter would facilitate the understanding of phase transformations between numerous known and hypothetical carbon allotropes from experimental point-ofview, and further stimulate the search of new applications of these phases, e.g., in electronics.

\section{Acknowledgments}

We acknowledge M. Czank and W. Depmeier for their assistance with Electron diffraction method according to DFG visiting grant. A.E. acknowledges a support from ISSC UB RAS (theme AAAAA19-119031890025-9). Visualizations of atomic structures and MD trajectories were performed using VMD software (www.ks.uiuc.edu/Research/vmd/) [39].

\section{References}

[1] Holmes J.D., Lyons D.M., Ziegler K.J. Supercritical fluid synthesis of metal and semiconductor nanomaterials. Chemistry Eur. J., 2003, 23, P. $2144-2150$

[2] Smolyar A.S., Barcholenko V.A., Kuts V.A., Gurin V.G., Archipov A.P., Maloshtan S.A., Razvadovsky N.A., Svyato V.P., Klimovich A.P. UA patent No 98084606. Bulletin No 6, 2002.

[3] Aust R.B., Drickamer H.C. Carbon: a new crystalline phase. Science, 1963, 140, P. 817; Deleted pattern No 18-311.

[4] Bandy F.P., Kasper J.S. Hexagonal diamond - a new form of carbon. J. Chem. Phys., 1967, 46, P. 3437-3446.

[5] Pokropivny V.V., Ivanovsky A.L. New nanoforms of carbon and boron nitride. Russ. Chem. Rev., 2008, 77, P. 837-874.

[6] Fedoseev D.V., Deryagin B.V., Varnin V.P., Vnukov S.P., Teremetskaya I.G., Polyanskaya N.D. Polymorphism in carbon-boron nitride systems. Dokl. Akad. Nauk SSSR, 1976, 228, P. 371-374.

[7] Smolyar A.S., Sozin Yu.I., Barholenko V.A., Maloshtan S.N., Kuts V.A., Gurin V.G., Arhipov A.P., Gerasimov A.Yu., Razvadovskii N.A., Titenko A.N.Fluid synthesis of carbon phases. J. Superhard Mater., 2002, 2, P. 79.

[8] Shterenberg I., Bogdanova S. Influence of nickel on graphitization of carbon materials at high-pressures and temperatures. Inorg. Mater., 1979, 15, P. 632-636.

[9] Shumilova T.G., Kablis G.N., Pushkarev E.V. Typomorphic features of graphite mineralization of probable alternative high-pressure sources of diamond: cubic graphite. Dokl. Earth Sciences, 2002, 387, P. 958-961.

[10] Wild R., Schellenbaum R. X-ray diffraction studies of mineral matter in North Dakota Lignite. Proc. North Dakota Acad. Sci., 1951, 5, P. 40-42.

[11] Pokropivny V.V., Pokropivny A.V. Structure of "cubic graphite": simple cubic fullerite C 24 . Phys. Solid State, 2004, 46, P. $392-394$.

[12] Pokropivny A.V. X-ray diffraction analysis of austite (cubic graphite), a novel carbon $\left[\mathrm{C}_{24}\right]$-LTA zeolite. Physics Low.-Dim. Struct., 2006, 2, P. 64-68.

[13] Pokropivny A.V., Volz S. C8 phase: supercubane, tetrahedral, BC-8 or carbon sodalite? Phys. Status Sol. B, 2012, 249, P. 1704-1708.

[14] Pokropivny A.V. Structure of the boron nitride E-phase: diamond lattice of $\mathrm{B}_{12} \mathrm{~N}_{12}$ fullerenes. Diam. Relat. Mat., 2006, 15, P. $1492-1495$.

[15] Pokropivny A.V., Volz S. Hybrid porous nanotube crystal networks for nanostructured device applications. J. Mater. Sci., 2013, 48, P. 29532960.

[16] Pokropivny V.V., Smolyar A.S., Pokropivny A.V. Fluid synthesis and structure of a new boron nitride polymorph-hyperdiamond fulborenite $\mathrm{B}_{12} \mathrm{~N}_{12}$ (E phase). Phys. Solid State, 2007, 49, P. 591-598.

[17] Baburin I.A., Proserpio D.M., Saleev V.A., Shipilova A.V. From zeolite nets to $\mathrm{sp}^{3}$ carbon allotropes: a topology-based multiscale theoretical study. Phys. Chem. Chem. Phys., 2015, 17, P. 1332-1338. 
[18] Hu M., Zhao Z., Tian F., Oganov A.R., Wang Q., Xiong M., Fan C., Wen B., He J., Yu D., Wang H.-T., Xu B., Tian Y. Compressed carbon nanotubes: a family of new multifunctional carbon allotropes. Sci. Rep., 2013, 3, 1331.

[19] Belenkov E.A., Brzhezinskaya M.M., Greshnyakov V.A. Novel carbon diamond-like phases LA5, LA7 and LA8. Diam. Relat. Mat., 2014, 50, P. 9-14.

[20] Belenkov E.A., Greshnyakov V.A. Diamond-like phases formed from fullerene-like clusters. Phys. Solid State, 2015, 57, P. $2331-2341$.

[21] Belenkov E.A., Brzhezinskaya M.M., Greshnyakov V.A. Crystalline structure and properties of diamond-like materials. Nanosystems: Physics Chemistry Mathematics, 2017, 8, P. 127-136.

[22] Belenkov E.A., Greshnyakov V.A. Modeling of phase transitions of graphites to diamond-like phases. Phys. Solid State, 2018, 60, P. 12941302.

[23] Greshnyakov V.A., Belenkov E.A. Structures of diamond-like phases. J. Exp. Theor. Physics, 2011, 113, P. 86-95.

[24] Kvashnina Y.A., Kvashnin D.G., Kvashin A.G., Sorokin P.B. New allotropic forms of carbon based on D-60 and D-20 fullerenes with specific mechanical characteristics. JETP Lett., 2017, 105, P. 419-425.

[25] Kvashnina Y.A., Kvashnin A.G., Popov M.Y., Kulnitskiy A.A., Perezhogin I.A., Tyukalova E.V., Chernozatonskii L.A., Sorokin P.B., Blank V.D. Toward ultra-incompressible carbon materials: computational simulation and experimental observation. J. Phys. Chem. Letters, 2015, 6, P. 2147-2152.

[26] Kvashnina Y.A., Kvashnin A.G., Sorokin P.B. Investigation of new superhard carbon allotropes with promising electronic properties. J. Appl. Phys., 2013, 114, P. 183708.

[27] Ordejon P., Artacho E., Soler J.M., Self-consistent order-N denisity-functional calculations for very large systems. Phys. Rev. B, 1996, 53, P. R10441.

[28] Soler J.M., Artacho E., Gale J.D., Garcia A., Junquera J., Ordejon P., Sanchez-Portal D. The SIESTA method for ab initio order-N materials simulation. J. Phys. Condens. Matter, 2002, 14, P. 2745-2780.

[29] Porezag D., Frauenheim T., Köhler T., Seifert G., Kaschner R., Construction of tight-binding-like potentials on the basis of density-functional theory: application to carbon. Phys. Rev. B, 1995, 51, P. 12947.

[30] Köster A.M., Flores R., Geudtner G., Goursot A., Heine T., Patchkovskii S., Reveles J.U., Vela A., Salahub D., deMon, Version 1.2; NRC: Ottawa, 2004.

[31] Caglioti G., Paoletti A., Ricci F.P. Choice of collimators for a crystal spectrometer for neutron diffraction. Nucl. Instr. Meth., 1958, 3, P. 223228.

[32] Baerlocher Ch., McCusker L.B., Olson D.H. Atlas of zeolite framework types. Elsevier, Amsterdam, 2007,404 p.

[33] http://www.ioffe.ru/SVA/NSM/Semicond/Diamond/

[34] Pugh S.F. Relations between the elastic moduli and plastic properties of polycrystalline pure metals. Phil. Mag., 1954, 45, P. 822-843.

[35] Zener C.M., Siegel S. Elasticity and anelasticity of metals. J. Phys. Chem., 1949, 53, P. 1468.

[36] Teter D.M. Computational alchemy: the search for new superhard materials. MRS Bull, 1998, 23, P. $22-27$.

[37] Chen X.Q., Niu H., Li D., Li Y. Modeling hardness of polycrystalline materials and bulk metallic glasses. Intermetallics, 2011, 19, P. 12751281.

[38] Shumilova T.G. Mineralogy of natural carbon (in Russian). Ural Branch of Russian Academy of sciences, Ekaterinburg, 2003, 316 p.

[39] Humphrey W., Dalke A., Schulten K., VMD: visual molecular dynamics. J. Mol. Graphics, 1996, 14, P. 33-38. 